\title{
ON DIFFERENTIAL HOPF ALGEBRAS
}

\author{
BY \\ WILLIAM BROWDER(1)
}

Differential Hopf algebras arise in several contexts in algebraic topology. The Bockstein spectral sequence of an $H$-space is one example that has been investigated by many authors $[3 ; 1 ; 7 ; 8]$. Borel [3] and Araki [1] proved algebraic theorems about the structure of differential Hopf algebras of special kinds. These special theorems enabled them to determine the odd torsion in the cohomology of the exceptional Lie groups.

If $X$ and $Y$ are $H$-spaces and $f: X \rightarrow Y$ is a fibre map which is multiplicative, then the spectral sequence of $f$ is a spectral sequence of Hopf algebras. This situation was first discussed by J. C. Moore [17], and later by the author $[5 ; 6]$. The techniques of [5] were later extended by the author (in unpublished work) to prove theorems about the homology and cohomology suspensions, i.e., when $X$ is the space of paths of the $H$-space $Y$. The proofs rested upon a general theorem about the structure of this spectral sequence. Some of these suspension theorems had been proved by Moore using a different spectral sequence of Hopf algebras [12].

In this paper we make a study of differential Hopf algebras, and prove general theorems on the structure of their homology. These theorems generalize the results of Borel and Araki. Applied to the case of multiplicative fibre maps, we obtain a general theorem about the structure of the spectral sequence (even in the nonacyclic case), which, in particular, yields simple proofs of the suspension theorems mentioned above. Applied to the Bockstein spectral sequence, we get information on torsion in $H$-spaces.

This study of differential Hopf algebras depends on two spectral sequences which may be defined in different circumstances. If one of them is defined, then the terms of that spectral sequence satisfy the conditions necessary for the other to be defined. Thus we get a spectral sequence for the term of the other spectral sequence $\left({ }^{2}\right)$, and this spectral sequence has a very simple form which makes it easy to calculate the form of its homology. Thus the structure of the limit

Received by the editors March 15, 1962.

(1) This research was supported in part by the National Science Foundation under Grant NSF-G15984.

(2) When this process is applied to the term of a spectral sequence, we find ourselves in the position of calculating a spectral sequence for the term of a spectral sequence for the term of a spectral sequence! 
term may be described very simply. The technique requires only that the differential Hopf algebra be either associative or coassociative. If it happens to be associative and commutative, then the structure of the limit term of the spectral sequence determines the algebraic structure of the homology to a large extent.

$\S \S 1,2$ and 3 develop the general theory; $\S \S 4,5$ and 6 are devoted to applications.

In $\$ 1$ we define the filtrations we use to obtain our spectral sequences, the first being that introduced in [16], the second being the dual filtration. In $\$ 2$ we discuss the "biprimitive" form of a Hopf algebra. In $\S 3$ we obtain spectral sequences for differential Hopf algebras in appropriate circumstances, and obtain our main tool, a spectral sequence of biprimitive Hopf algebras, starting with the biprimitive form of $A$, with limit the biprimitive form of $H(A)$. In $\S 4$ we apply the results to the Bockstein spectral sequence of an $H$-space, and in $\S 5$ to multiplicative fibre maps, where we obtain various transgression theorems. In $\S 6$ we apply these results to Lie groups, or similar $H$-spaces, and get theorems about existence of torsion in their homology, and other theorems.

We refer to [16] for the theory of Hopf algebras, and we employ the results of [16] freely.

Much of this work overlaps with unpublished work of J. C. Moore on differential Hopf algebras. I am indebted to him for many conversations.

1. Filtrations of Hopf algebras. We shall assume that all graded modules $\boldsymbol{M}$ considered have $M_{i}$ finitely generated over $K$ for all $i, M_{i}=0$ for $i<0, M_{0}=K$, the ground field, which will always be taken as $Z_{p}, p$ prime, or the rationals, $Z_{0}$.

Definition. An algebra $A$ over a field $K$ is graded $K$-module $A=\sum_{i \geqq 0} A_{i}$ and a map of graded modules $\phi: A \otimes A \rightarrow A\left(\phi\left(A_{i} \otimes A_{j}\right) \subseteq A_{i+j}\right)$ such that there exists a unit $1 \in A_{0}(\phi(1 \otimes x)=\phi(x \otimes 1)=x$ for any $x \in A)$.

Let $A$ be an algebra, and let $\bar{A}=$ positive dimensional elements of $A$. Define a decreasing filtration $\left\{F^{q} A\right\}$ of $A$ as follows:

$$
F^{0} A=A, \quad F^{n+1} A=\phi\left(\bar{A} \otimes F^{n} A\right) .
$$

We set $E_{0}^{q}(A)=F^{q}(A) / F^{q+1}(A)$ and $E_{0}=\Sigma_{q} E_{0}^{q}$. $E_{0}^{q}$ is graded and we set $E_{0}^{q, l}=(q+l)$ dimensional elements of $E_{0}^{q}$, so that $E_{0}=\Sigma_{q, l} E_{0}^{q, l}$. It is easy to check that $E_{0}^{0}=K$ and $E_{0}^{q, l}=0$ for $l<0$.

For $x \in F^{q}, x \notin F^{q+1}$, define $\bar{x}=\{x\}$ in $F^{q} / F^{q+1}$. For every $x \in A$ there exists such a $q$, so $\bar{x}$ is defined for each $x$.

If $A$ is an associative algebra, we may define the structure of an algebra on $E_{0}$ by $\bar{x} \cdot \bar{y}=\overline{x y}$.

Proposition 1.1. Let $A$ and $B$ be associative algebras.

(i) $E_{0}(A)$ and $E_{0}(B)$ are associative algebras.

(ii) $E_{0}(A \otimes B)=E_{0}(A) \otimes E_{0}(B)$, where the tensor product of two algebras is made an algebra by the rule $(a \otimes b) \cdot(c \otimes d)=(-1)^{p q} a c \otimes b d, \operatorname{dim} b=p$, $\operatorname{dim} c=q$. 
(iii) $A$ homomorphism $f: A \rightarrow B$ induces a homomorphism $f_{0}: E_{0}(A) \rightarrow E_{0}(B)$.

(iv) $E_{0}^{1}=$ indecomposable elements of $E_{0}$, i.e., no element of $E_{0}^{1}$ is a product and $E_{0}^{1}$ generates $E_{0}$.

Proof. (i) $F^{p} A \cdot F^{q} A \subset F^{p+q} A$ since $A$ is associative and thus the multiplication in $E_{0}(A)$ is well defined and $E_{0}(A)$ is an algebra, obviously associative.

(ii) $\overline{A \otimes B}=\bar{A} \otimes B+A \otimes \bar{B}$. Hence if $F^{p}(A \otimes B)=\sum_{i+j={ }_{p}} F^{i} A \otimes F^{j} B$, then

$$
\begin{aligned}
F^{p+1}(A \otimes B) & =(\bar{A} \otimes B+A \otimes \ddot{B}) F^{p}(A \otimes B) \\
& =\sum_{i+j=p+1} F^{i} A \otimes F^{j} B .
\end{aligned}
$$

Hence $E_{0}^{p}(A \otimes B)=\sum_{i+j=p} E_{0}^{i}(A) \otimes E_{0}^{j}(B)$ and (ii) follows.

(iii) and (iv) are obvious.

Definition. A coalgebra $A$ over $K$ is a graded $K$-module $A$ together with a map $\psi: A \rightarrow A \otimes A$ of graded modules such that, for $x \in \bar{A}, \psi x=x \otimes 1+1 \otimes x$ $+\sum x_{i} \otimes x_{i}^{\prime}$, where $1 \in A_{0}=K, x_{i}, x_{i}^{\prime} \in A . \quad A$ is coassociative if $(\psi \otimes 1) \circ \psi$ $=(1 \otimes \psi) \circ \psi$.

Let $A$ be a coalgebra over $K$, and let $\rho: A \rightarrow \bar{A}$ be the natural projection, $i: \bar{A} \subset A$. Define $\bar{\psi}: \bar{A} \rightarrow \bar{A} \otimes \bar{A}$, by $\bar{\psi}=(\rho \otimes \rho) \circ \psi \circ i$. Define $\bar{\psi}_{n}: A \rightarrow \bar{A} \otimes \cdots \otimes \bar{A}$ $(n+1$ times $)$ by $\bar{\psi}_{0}=\rho$ and $\bar{\psi}_{n+1}=(\bar{\psi} \otimes 1 \otimes \cdots \otimes 1) \circ \bar{\psi}_{n}$, and let $G^{p} A=$ kernel $\bar{\psi}_{p}$. Then $G^{0} A=A_{0}$ and $\left\{G^{p} A\right\}$ is an increasing filtration of $A . G^{p} A \subseteq G^{p+1} A$, $\bigcup_{p} G^{p} A=A$. Set ${ }_{0} E^{p}(A)=G^{p} A / G^{p-1} A$ and ${ }_{0} E(A)=\sum_{p} E^{p}(A)$.

The dual $A^{*}=\operatorname{Hom}(A, K)$ of the coalgebra $A$ is an algebra, and it is easy to see that $F^{p+1} A^{*}=$ annihilator of $G^{p} A$, i.e., $F^{p+1}$ and $G^{p}$ are dual filtrations.

If $A$ is coassociative, we may define a coalgebra structure in ${ }_{0} E$ by $\Psi_{0}(\bar{x})=(\overline{\psi x})$.

Proposition 1.2. Let $A$ and $B$ be coassociative coalgebras.

(i) ${ }_{0} E(A),{ }_{0} E(B)$ are coassociative coalgebras.

(ii) ${ }_{0} E(A \otimes B)={ }_{0} E(A) \otimes{ }_{0} E(B)$ as coalgebras.

(iii) $A$ homomorphism $f: A \rightarrow B$ of coalgebras induces a homomorphism of coalgebras $f_{0}:{ }_{0} E(A) \rightarrow{ }_{0} E(B)$.

(iv) $P\left({ }_{0} E(A)\right)={ }_{0} E^{1}(A)\left(P\left({ }_{0} E(A)\right)=\right.$ the primitive elements of ${ }_{0} E(A)$, i.e., the elements $x$ such that $\left.\Psi_{0} x=x \otimes 1+1 \otimes x\right)$.

The proof is similar (dual) to the proof of Proposition 2.1.

Definition. A Hopf algebra over a field $K$ is a (graded) module $A$ over $K$ such that $A_{0}=K, A_{i}=0$ for $i<0$, equipped with maps $\psi: A \rightarrow A \otimes A$, and $\phi: A \otimes A \rightarrow A$ such that

(1) $\phi$ makes $A$ a graded algebra over $K$,

(2) $\psi(x)=x \otimes 1+1 \otimes x+\sum x_{i} \otimes x_{i}^{\prime}$ for $x \in A_{m}, m>0$, where $0<\operatorname{dim} x_{i}<m$, $0<\operatorname{dim} x_{i}^{\prime}<m$ ( $\psi$ makes $A$ a graded coalgebra).

(3) $\psi$ is a map of algebras, where $A \otimes A$ is made an algebra by defining

$$
(a \otimes b) \cdot(c \otimes d)=(-1)^{p q} \phi(a \otimes c) \otimes \phi(b \otimes \mid d),
$$

where $b \in A_{p}, c \in A_{q}$. 
If $A$ is an associative Hopf algebra, then $\psi: A \rightarrow A \otimes A$ is a map of algebras which induces a Hopf algebra structure in $E_{0}(A)$. If $A$ is a coassociative Hopf algebra, the product $\phi: A \otimes A \rightarrow A$ is a map of coalgebras, which induces a Hopf algebra structure in ${ }_{0} E(A)$.

Proposition 1.3. (i) If $A$ is an associative Hopf algebra, then $E_{0}(A)$ is a Hopf algebra and $E_{0}^{1}(A) \subset P\left(E_{0}(A)\right)$, so that $E_{0}(A)$ is primitively generated.

(ii) If $A$ is a coassociative Hopf algebra over $Z_{p}$, then ${ }_{0} E(A)$ is a Hopf algebra, ${ }_{0} E(A)$ is associative, commutative and $\xi\left({ }_{0} \overline{E(A)}\right)=0 \quad\left(\right.$ where $\left.\xi(x)=x^{p}\right)$.

(iii) If $A$ is an associative Hopf algebra, then $E_{0}(A)$ and ${ }_{0} E\left(A^{*}\right)$ are dual Hopf algebras.

Proof. (i) $\Psi_{0}: E_{0} \rightarrow E_{0} \otimes E_{0}$ preserves filtration, and since $E_{0}^{0}=K$, $E_{0}^{1} \subset P\left(E_{0}\right) . E_{0}^{1}$ generates $E_{0}$ by (1.1(iv)).

(iii) follows easily from the fact that the two filtrations are dual. Then (ii) follows from (i) and (iii), together with [16, Theorem 4.9]: A Hopf algebra $A$ over $Z_{p}$ is primitively generated if and only if the dual Hopf algebra $A^{*}=\operatorname{Hom}\left(A, Z_{p}\right.$, is commutative, associative, and $\xi\left(\overline{A^{*}}\right)=0$, (see theorem 2 below).

Proposition 1.4. (i) If $A$ is an associative, commutative Hopf algebra over $Z_{p}$, then $E_{0}(A) \cong A$ as algebras.

(ii) If $A$ is a coassociative, cocommutative Hopf algebra over $Z_{p}$, then ${ }_{0} E(A) \cong A$, as coalgebras.

Proof. (i) follows from Proposition 1.1 (ii) and Borel's theorem on the structure of Hopf algebras [2, Theorem 6.1] and [16]. (ii) is simply the dual statement of (i).

\section{Biprimitive forms.}

Definition. A Hopf algebra $A$ is said to be primitive if there exists a set of primitive generators for $A$. $A$ will be called coprimitive if the primitive elements of $A$ are indecomposable. We shall say $A$ is biprimitive if it is both primitive and coprimitive.

It is easy to see that $A$ coprimitive is equivalent to $A^{*}$ primitive ( $A^{*}=$ the dual Hopf algebra).

We recall a result of Milnor and Moore [16, Theorems 4.8 and 4.9] (we include a proof in $§ 7)$ :

THEOREM 2.1. A Hopf algebra $A$ over $Z_{p}, p \neq 0$, is coprimitive if and only if $A$ is associative, commutative and all pth powers of elements of $\bar{A}$ are zero. $A$ Hopf algebratover the rationals is coprimitive if and only if its multiplication is associative and commutative.

Corollary 2.2. If $A$ is a coprimitive Hopf algebra over $Z_{p}$, then the integers $r_{i}=\operatorname{rank} A_{i}$ (as a $Z_{p}$-module) determine the algebra $A$ completely. In other 
words if $A$ and $B$ are coprimitive Hopf algebras over $Z_{p}$, and if rank $A_{i}=\operatorname{rank} B_{i}$ for all $i$, then $A$ is isomorphic to $B$ as algebras.

Proof. By Borel's Theorem [2, Theorem 6.1] and Theorem 2.1, $A \cong \bigotimes_{i} A\left(x_{i}\right)$ as an algebra, where $\left\{x_{i}\right\}$ are a set of generators for $A$ and $A\left(x_{i}\right)=Z_{p}\left[x_{i}\right] /\left(x_{i}^{p}\right)$ when $\operatorname{dim} x_{i}$ is even, $A\left(x_{i}\right)=\wedge\left(x_{i}\right)=$ exterior algebra on $x_{i}$, when $\operatorname{dim} x_{i}$ is odd (it is understood that $x^{p}=0$ if $p=0$ ). Hence the dimensions of the generators $x_{i}$ determine $A$ completely as an algebra.

Let us assume by induction that $r_{i}, i<n$, determines $A$ completely in dimensions less than $n$. Then rank $D_{n}$, where $D_{n}=$ decomposable elements of dimension $n$, is determined. Then, $r_{n}-\left(\right.$ rank $\left.D_{n}\right)=$ number of generators of dimension $n$, which proves the corollary.

Corollary 2.3. If $A$ is a biprimitive Hopf algebra, then the numbers $r_{i}$ $=$ rank $A_{i}$ determine the Hopf algebra A completely.

Proof. If $A$ is primitive, the generators of $A$ may be chosen primitive which determines the diagonal map of $A$.

Definition. Let $A$ be a Hopf algebra, $B$ a biprimitive Hopf algebra. $B$ is called a biprimitive form of $A$ if $\operatorname{rank} A_{i}=\operatorname{rank} B_{i}$ for all $i$.

Corollary 2.4. Any two biprimitive forms of a Hopf algebra are isomorphic (as Hopf algebras).

If $A$ is associative, then $E_{0}(A)$ is primitive by Proposition 1.3(i). If $A$ is coassociative, then ${ }_{0} E(A)$ is coprimitive by Proposition 1.3(ii). On the other hand a primitive Hopf algebra is coassociative, and a coprimitive Hopf algebra is associative.

LEMMA 2.5. If $A$ is primitive, then ${ }_{0} E(A)$ is biprimitive. If $A$ is coprimitive then $E_{0}(A)$ is biprimitive.

Proof. The statements are dual so that it suffices to prove one of them. We will prove the second. That $A$ is coprimitive is equivalent (by Theorem 2.1) to $A$ being associative, commutative, and having $p$ th powers of positive dimensional elements zero. By Proposition 1.4 (i) $E_{0}(A) \cong A$ as an algebra so that $E_{0}(A)$ has these properties and $E_{0}(A)$ is also coprimitive. But $E_{0}(A)$ is primitive by Proposition 1.3(i), so that $E_{0}(A)$ is biprimitive.

COROLlary 2.6. If $A$ is an associative Hopf algebra, then ${ }_{0} E\left(E_{0}(A)\right)$ is a biprimitive form of $A$. If $A$ is a coassociative Hopf algebra, then $E_{0}\left({ }_{0} E(A)\right)$ is a biprimitive form of $A$.

If $A$ is associative and commutative by Borel's Theorem $E_{0}(A) \cong A$ as an algebra.

If $B$ is associative, commutative and primitively generated, over $Z_{p}, p \neq 0$, it is easy to show that ${ }_{0} E(B)$ is obtained from $B$ simply by truncatirg each 
polynomial ring to height $p$ and introducing new generators for each $p$ th power. Explicitly, if $B=\bigotimes_{i} B_{i}$ where $B_{i}$ is a Hopf algebra on one generator, then

and

$$
{ }_{0} E(B)=\bigotimes_{i} E\left(B_{i}\right)
$$

while

$$
{ }_{0} E\left(\Lambda\left(x_{i}\right)\right) \cong \Lambda\left(x_{i}\right)
$$

$$
{ }_{0} E\left(Z_{p}\left[x_{i}\right] / x_{i}^{p^{s}}\right)=\bigotimes_{k=1}^{f} Z_{p}\left[\xi_{k}\right] / \xi_{k}^{p}
$$

where $x_{i}^{p^{k-1}}$ is a representative of $\xi_{k}$.

Hence we get the following theorem which will be used repeatedly to investigate a Hopf algebra via its biprimitive form.

THEOREM 2.7. Let $A$ be an associative, commutative Hopf algebra over $Z_{p}$. As an algebra, the biprimitive form of $A$ is obtained from $A$ by replacing tensor product factors of the form $Z_{p}[x] /\left(x^{p^{f}}\right)$ by $\bigotimes_{i=1}^{f} Z_{p}\left[\xi_{i}\right] /\left(\xi_{i}^{p}\right)$ where $x^{p^{i-1}}$ represents $\xi_{i}$.

If $p=0$, we are to take $x^{p}=0$. That is, the theorem says that $A$ is isomorphic as an algebra to its biprimitive form, if we have rational coefficients.

\section{Differential Hopf algebras.}

Definition. A differential Hopf algebra is a Hopf algebra $A$ with a differential $d\left(d^{2}=0\right)$ such that the product and diagonal maps $\phi: A \otimes A \rightarrow A$ and $\psi: A \rightarrow A \otimes A$ are maps of differential modules (where $A \otimes A$ has the usual tensor product differential, $d(a \otimes b)=(d a) \otimes b+(-1)^{p} a \otimes d b$, if $\left.a \in A_{p}\right)$.

If $A$ is an associative differential Hopf algebra, then $d$ preserves the filtration $F^{p}$ of $\S 1$ of the algebra $A$, i.e., $d\left(F^{p} A\right) \subseteq F^{p} A$.

THEOREM 3.1. Let $A$ be an associative differential Hopf algebra. Then associated with the filtration $F^{p} A$ we get a spectral sequence of associative, primitive Hopf algebras $E_{r}, 0 \leqq r \leqq \infty\left(E_{0}=E_{0}(A)\right.$ as in $\left.\$ 1\right)$.

Proof. If $A$ is associative, then $\phi: A \otimes A \rightarrow A$ is a map of filtered modules (which induces the algebra structure of $E_{0}(A)$ ). Hence $\phi$ is a map of filtered differential modules and hence induces a map of spectral sequences $\phi_{r}: E_{r}(A \otimes A) \rightarrow E_{r}(A), 0 \leqq r \leqq \infty$. But $E_{r}(A \otimes A)=E_{r}(A) \otimes E_{r}(A)$ so that $\phi_{r}$ makes $E_{r}(A)$ an algebra. Since $\psi: A \rightarrow A \otimes A$ is a homomorphism of differential algebras, it induces $\psi_{r}: E_{r}(A) \rightarrow E_{r}(A) \otimes E_{r}(A)$ which makes $E_{r}(A)$ a Hopf algebra. Since $\phi_{r}$ and $\psi_{r}$ commute with $d_{r}$ (being maps of spectral sequences), it follows that $\left\{E_{r}\right\}$ is a spectral sequence of differential Hopf algebras.

Since $\phi$ is associative, $E_{0}(A)$ is associative (see Proposition 1.1), and primitive see Proposition 1.3(i)). Then the following lemma will complete the proof of the theorem (by induction). 
Lemma 3.2. Let $A$ be a differential Hopf algebra over $Z_{p}$. (a) If $A$ is associative, then $H(A)$ is associative. (b) If $A$ is primitive, then $H(A)$ is primitive. (c) If $A$ is coprimitive, then $H(A)$ is coprimitive.

Proof. (a) is obvious, while (b) and (c) are dual.

To prove (c) we use Theorem 2.1 , so it suffices to prove that $H(A)$ is commutative, associative and that all $p$ th powers of positive dimensional elements of $H(A)$ are zero. But these properties all follow easily from the corresponding properties of $A$, so that the lemma follows.

The dual theorem below may be obtained directly from Theorem 3.1 by duality.

THEOREM 3.3. Let $A$ be a coassociative differential Hopf algebra. Then associated with the filtration $G^{q}$, we get a spectral sequence of coassociative, coprimitive Hopf algebras, ${ }_{r} E, 0 \leqq r \leqq \infty\left({ }_{0} E={ }_{0} E(A)\right.$ as in $\left.\S 1\right)$.

Now a primitive Hopf algebra $A$ is coassociative, and a coprimitive Hopf algebra $B$ is associative (see Theorem 2.1). Hence we may use the filtration $G^{q}$ on a primitive $A$, or the filtration $F^{p}$ on a coprimitive $B$.

Lemma 3.4. Let $A$ be a primitive differential Hopf algebra. Then ${ }_{\infty} E(A)$ is a biprimitive form of $H(A)$.

Proof. ${ }_{\infty} E(A)$ is the graded module associated with a filtration of $H(A)$, by the usual theory of spectrals sequences (see [11]). Hence $\operatorname{rank}\left({ }_{\infty} E(A)\right)^{q}=\operatorname{rank} H^{q}(A)$ for each $q$ (total degree $q$ in ${ }_{\infty} E(A)$ ). Then it remains to show ${ }_{\infty} E(A)$ is biprimitive. By Lemma 2.5, ${ }_{0} E(A)$ is biprimitive. But it follows from Lemma 3.2 that if ${ }_{r} E$ is biprimitive, then ${ }_{r+1} E=H\left({ }_{r} E\right)$ is biprimitive, and the lemma follows by induction. (Note that for any given total degree $q,\left({ }_{\infty} E\right)^{q}=\left({ }_{q+1} E\right)^{q}$, so that it involves only a finite number of steps to prove the theorem in any given dimension, and thus the induction is valid.)

We immediately get the dual:

Lemma 3.5. Let $A$ be a coprimitive differential Hopf algebra. Then $E_{\infty}(A)$ is a biprimitive form of $H(A)$.

Then in the spectral sequence $E_{r}(A)$ for an associative differential Hopf algebra $A$, we may take the spectral sequence ${ }_{s} E$ for each term.

LemMa 3.6. Let $A$ be an associative differential Hopf algebra. Then the spectral sequence ${ }_{s} E\left(E_{r}(A)\right)$ converges to ${ }_{0} E\left(E_{r+1}(A)\right) \quad($ as $s \rightarrow \infty)$, i.e., ${ }_{\infty} E\left(E_{r}(A)\right) \cong{ }_{0} E\left(E_{r+1}(A)\right)$, as Hopf algebras.

Proof. $E_{r}(A)$ is primitive, by Theorem 3.1. Hence ${ }_{\infty} E\left(E_{r}(A)\right)$ is a biprimitive form of $H\left(E_{r}(A)\right)=E_{r+1}(A)$ by Lemma 3.4. Since $E_{r+1}(A)$ is primitive, ${ }_{0} E\left(E_{r+1}(A)\right)$ is also a biprimitive form of $E_{r+1}(A)$ so that ${ }_{\infty} E\left(E_{r}(A)\right) \cong{ }_{0} E\left(E_{r+1}(A)\right)$ as Hopf algebras, by Corollary 2.3. 
We get by duality:

LEMMA 3.7. Let $A$ be a coassociative differential Hopf algebra. Then the spectral sequence $E_{s}\left(r_{r}(A)\right)$ converges to $E_{0}\left({ }_{r+1} E(A)\right) \quad($ as $s \rightarrow \infty)$, i.e., $E_{\infty}\left({ }_{r} E(A)\right) \cong E_{0}\left({ }_{r+1} E(A)\right)$ as Hopf algebras.

Now we come to our main result.

THEOREM 3.8. Let $A$ be a differential Hopf algebra, and suppose $A$ is either associative or coassociative. Then there exists a spectral sequence of biprimitive Hopf algebras with the first term the biprimitive form of $A$ and last term the biprimitive form of $H(A)$.

We shall refer to this spectral sequence as the biprimitive spectral sequence of $A$.

(Note that by spectral sequence we mean $E_{r}$ with $0 \leqq r \leqq \alpha$, where $\alpha$ is some ordinal, rather than the usual convention where $\alpha=\omega$ the first limit ordinal. In the proof below $\alpha=\omega \cdot \omega$.)

Proof. Let $A$ be associative. Then in the spectral sequence $E\left(E_{r}(A)\right)$, ${ }_{\infty} E\left(E_{r}(A)\right)={ }_{0} E\left(E_{r+1}(A)\right)$ by Lemma 3.6, so that ${ }_{0} E\left(E_{r}(A)\right),{ }_{1} E\left(E_{r}(A)\right), \cdots$, ${ }_{\infty} E\left(E_{r}(A)\right)={ }_{0} E\left(E_{r+1}(A)\right),{ }_{1} E\left(E_{r+1}(A)\right), \cdots$ is a spectral sequence. Thus juxtaposition of the spectral sequences $\left.\left\{{ }_{s} E\left(E_{0}(A)\right)\right\},{ }_{s} E\left(E_{1}(A)\right)\right\}, \cdots$ is the spectral sequence required.

If $A$ is coassociative, we take $\left\{E_{s}\left({ }_{0} E(A)\right)\right\},\left\{E_{s}\left({ }_{1} E(A)\right)\right\}, \cdots$, using Lemma 3.7, which completes the proof.

Thus questions about the biprimitive form of $H(A)$ can be reduced by Theorem 3.8 to questions about the homology of biprimitive differential Hopf algebras. These are described below.

A differential Hopf algebra $B$ is said to be an elementary differential Hopf algebra of type $\mathrm{I}$ if $B=(x) \otimes Z_{p}[y] /\left(y^{p}\right), x, y$ primitive, $d x=y, \operatorname{dim} x$ odd, $\operatorname{dim} y$ even. It is of type II if $d y=x$, everything else as in type I. Such algebras are clearly biprimitive.

A biprimitive differential Hopf algebra $A$ is said to be of type I (resp. II) if $A \cong \bigotimes_{i} B_{i}$ as differential Hopf algebras, where $B_{i}$ is an elementary differential Hopf algebra of type I (resp. II) for each $i$. $A$ is said to be of type III if $d \equiv 0$.

THEOREM 3.9. Let $A$ be a biprimitive differential Hopf algebra over $Z_{p}$. Then $A$ is the tensor product of differential Hopf algebras of types I, II, and III (as a differential Hopf algebra). Explicitly $A \cong \bigotimes_{i} K_{i} \otimes \bigotimes_{j} M_{j} \otimes Q$ where

$$
\begin{array}{ll}
K_{i}=\wedge\left(x_{i}\right) \otimes Z_{p}\left[y_{i}\right] /\left(y_{i}^{p}\right), & d x_{i}=y_{i}, x_{i}, y_{i} \text { primitive, } \\
M_{j}=\wedge\left(\xi_{j}\right) \otimes Z_{p}\left[\eta_{j}\right] /\left(\eta_{j}^{p}\right), & d \eta_{j}=\xi_{j}, \xi_{j}, \eta_{j} \text { primitive, }
\end{array}
$$

$\operatorname{dim} x_{i}, \xi_{j}$ odd, $\operatorname{dim} y_{i}, \eta_{j}$ even, $Q$ a biprimitive Hopf algebra with $d \equiv 0$.

Proof. Since $A$ is biprimitive, any basis of $P(A)$, the primitive elements of $A$, will be a set of generators of $A$ satisfying the hypothesis of Borel's Theorem. 
Then as a Hopf algebra, $A$ will be the tensor product of the algebras generated by each of these generators, truncated polynomial algebras of height $p$ for even dimensional generators, exterior algebras for odd dimensional generators.

Then we may find modules $F, G, J$, with $P(A)=F+G+J, d(F)=G$ and $d \mid F$ is an isomorphism of $F$ with $G, d(J)=0$. If $M_{+}\left(M_{-}\right)$denotes the even (odd) dimensional elements of $M$ ( $M$ any module), then $\bigotimes_{i} K_{i}$ is generated by $F_{-}+G_{+}, \bigotimes_{j} M_{j}$ is generated by $F_{+}+G_{-}, Q$ is generated by $J$.

THEOREM 3.10. (a) Let $K=\wedge(x) \otimes Z_{p}[y] /\left(y^{p}\right)$ with $d x=y, \operatorname{dim} x$ odd. Then $H(K)=\wedge\left(\left\{x y^{p-1}\right\}\right)$, if $p \neq 0 ; H(K)=Z_{0}$ if $p=0$.

(b) Let $M=\wedge(\xi) \otimes Z_{p}[\eta] /\left(\eta^{p}\right)$ with $d \eta=\xi$, $\operatorname{dim} \xi$ odd. Then $H(M)$ $=\wedge\left(\left\{\xi \eta^{p-1}\right\}\right)$, if $p \neq 0 ; H(M)=Z_{0}$ if $p=0$.

(c) If $d(Q)=0, H(Q)=Q$.

This theorem follows immediately by direct computation.

THEOREM 3.11. Let $A$ be a differential Hopf algebra which is either associative or coassociative. If $A$ is finite dimensional, i.e., $A_{i}=0$ for large $i$, then the biprimitive form of $H(A)$ has the same number of odd dimensional generators as the biprimitive form of $A$.

Proof. This follows from Theorems 3.8, 3.9, and 3.10. For we need only verify this in differential Hopf algebras of types I, II, and III where it is obvious. Thus the only way an odd dimensional generator may disappear (e.g., in ${ }_{s} E\left(E_{r}(A)\right)$ ) is by a process of replacement by new odd dimensional generators, whose dimension goes to $\infty$, which contradicts the finite dimensionality of $A$. Note that if the coefficients are the rationals $Z_{0}$, the finite dimensionality implies that $d(A)=0$.

Corollary 3.12. Let $A$ be an associative, commutative, differential Hopf algebra. If $A$ is finite dimensional, then $H(A)$ has the same number of odd dimensional generators as $A$.

Proof. This follows immediately from Theorem 3.11 and Theorem 2.7.

This Corollary is due to J. C. Moore (unpublished).

Further we have:

THEOREM 3.13. Let $A$ be a differential Hopf algebra which is either associative or coassociative. Suppose $A_{m} \neq 0$ and $A_{i}=0$ for $i>m$. Then $A_{m} \cong Z_{p}$ $\cong H_{m}(A)$ and $H_{i}(A)=0$ for $i>m$.

Proof. This is easy to verify for a biprimitive differential Hopf algebra, so that the theorem follows by applying the biprimitive spectral sequence of $A$.

4. Applications to the Bockstein spectral sequence. In this section we apply our results to studying the Bockstein spectral sequence of an $H$-space. As a con- 
sequence we get a theorem describing the Bockstein spectral sequence of a finite dimensional $H$-space.

The exact coefficient sequence $0 \rightarrow Z \rightarrow Z \rightarrow Z_{p} \rightarrow 0$ ( $p$ a prime, as usual) leads to a homology (or cohomology) exact couple:<smiles>[R14][14CH]([R14])[14CH3]</smiles>

The spectral sequence of this exact couple is called the homology (or cohomology) Bockstein spectral sequence $\left\{B^{k}\right\}$ (or $\left\{B_{k}\right\}$ ) of $X, \bmod p$. In case $X$ is an $H$-space, and $H_{i}(X)$ is finitely generated for each $i$, the homology and cohomology Bockstein spectral sequences are dual spectral sequences of Hopf algebras. (For details and a thorough discussion of the Bockstein spectral sequences see [7].) We denote by $\beta_{k}$ and $\beta^{k}$ the differentials in $B_{k}$ and $B^{k}$, respectively.

Since the Bockstein spectral sequence in cohomology $\bmod p$ of an $\mathrm{H}$-space is a spectral sequence of associative, commutative Hopf algebras, the discussion of $\$ 3$ applies. Thus for each term of the spectral sequence we have a spectral sequence of biprimitive Hopf algebras. Using the results of [7], we may describe the spectral sequence more precisely in the case of a finite dimensional $H$-space.

THEOREM 4.1. Let $X$ be a connected $H$-space with $H_{i}(X)$ finitely generated for each $i$, and $H_{j}\left(X ; Z_{p}\right)=0$ for $j>n$. Then in ${ }_{s} E\left(E_{r}\left(B_{k}\right)\right)\left(B_{k}=\right.$ the kth term of the cohomology Bockstein spectral sequence mod $p$ of $X)$, all even dimensional primitive elements are cycles.

This theorem is dual to the following theorem and equivalent to it.

THEOREM 4.2. Let $X$ be as in Theorem $4.1, B^{k}=$ the Bockstein spectral sequence in homology mod $p$ of $X, B^{k}=\left(B_{k}\right)^{*}$. Then all odd dimensional primitive elements of $E_{m}\left({ }_{l} E\left(B^{k}\right)\right)$ are cycles.

To show Theorem 4.1 is equivalent to Theorem 4.2 , we note that $B^{k}=\left(B_{k}\right)^{*}$, ${ }_{r} E\left(A^{*}\right)=\left(E_{r}(A)\right)^{*}, E_{m}\left(B^{*}\right)=\left({ }_{m} E(B)\right)^{*}$ (as differential Hopf algebras in each case). It follows that $E_{m}\left({ }_{l} E\left(B^{k}\right)\right)=\left({ }_{m} E\left(E_{l}\left(B_{k}\right)\right)\right)^{*}$.

The following lemma follows immediately from the definitions:

LEMMA 4.3. If $B$ is biprimitive, then $P\left(B^{*}\right)=(P(B))^{*}$.

For $Q\left(B^{*}\right)=(P(B))^{*}$, and the map of $P\left(B^{*}\right)$ into $Q\left(B^{*}\right)$ is an isomorphism.

Let $D={ }_{s} E\left(E_{r}\left(B_{k}\right)\right)$. Then Theorem 4.1 asserts that $d: P\left(D^{2 n}\right) \rightarrow P\left(D^{2 n+1}\right)$ is zero. By virtue of Lemma 4.3 this is equivalent to $d^{*}: P\left(D_{2 n+1}\right) \rightarrow P\left(D_{2 n}\right)$ being zero, which is Theorem 4.2 .

We shall prove a slightly stronger theorem which implies Theorem 4.1.

THEOREM 4.4. Let $X$ be a connected $H$-space with $H_{i}(X)$ finitely generated for each $i$ and $H_{j}\left(X ; Z_{p}\right)=0$ for $j>n$ (for some $n$ ). Then in $E_{r}\left(B_{k}\right)$, all even dimensional primitive elements are cycles under $d_{r}$. 
Proof of Theorem 4.4. From the results of $\$ 2$, it follows that the even dimensional primitive elements of $E_{0}\left(B_{k}\right)$ are represented by even dimensional generators and their iterated $p$ th powers in $B_{k}$. Since $\beta_{k}$, the differential in $B_{k}$, is a derivation and $B_{k}$ is associative and commutative, the $p$ th powers are cycles in $B_{k}$. Hence the primitive elements in $E_{0}\left(B_{k}\right)$ corresponding to $p$ th powers in $B_{k}$ are permanent cycles in $E_{r}\left(B_{k}\right)$. Therefore, to prove Theorem 4.4, it suffices to consider primitive elements of $E_{r}\left(B_{k}\right)$ which are represented by elements $x \in B_{k}^{2 m}$ which are indecomposable.

Lemma 4.5. $\beta_{k}\left(B_{k}^{2 m}\right) \subseteq \beta_{k}(D)$, where $D=$ the decomposable elements of $B_{k}^{2 m}$.

Proof. If $\beta_{k} x \notin \beta_{k}(D)$, then there is an element $\bar{y} \in B_{2 m+1}^{k}$, with $\bar{y}\left(\beta_{k} x\right) \neq 0$ and $\bar{y}\left(\beta_{k} D\right)=0$. Hence $\left(\beta^{k} \bar{y}\right)(x) \neq 0$ and $\left(\beta^{k} \bar{y}\right)(D)=0$, i.e., $\beta^{k} \bar{y} \neq 0$ and is primitive, which by [7, Theorem 6.1], contradicts the hypothesis that $H_{*}\left(X ; Z_{p}\right)$ is a finite dimensional $Z_{p}$ module $\left(H_{j}\left(X ; Z_{p}\right)=0\right.$ for $\left.j>n\right)$. Hence $\beta_{k} x \in \beta_{k}(D)$.

Let $x \in B_{k}^{2 m}$ be an indecomposable element representing a primitive element $z \in E_{0}^{1}\left(B_{k}\right)=F^{1}\left(B_{k}\right) / F^{2}\left(B_{k}\right)$. We shall show that $z$ is a permanent cycle in $E_{r}\left(B_{k}\right)$ which will prove the theorem. If $\beta_{k} x \neq 0$, then $\beta_{k} x=\beta_{k} w$, where $w \in D=F^{2}$ by Lemma 4.5. Then $z=\{x\}=\{x-w\}$ in $E_{0}^{1}=F^{1} / F^{2}$ and $\beta_{k}(x-w)=0$. Hence $z$ is a permanent cycle. q.e.d.

THEOREM 4.6. Let $X$ be an $H$-space, with $H_{*}(X)$ finitely generated. Then there is spectral sequence of biprimitive Hopf algebras whose first term is the biprimitive form of $H^{*}\left(X ; Z_{p}\right)$ and whose last term is the biprimitive form of $B_{\infty}=\left(H^{*}(X) /\right.$ torsion $) \otimes Z_{p}$. Each term in the biprimitive spectral sequence is a tensor product of differential Hopf algebras of types I and III.

Proof. The spectral sequence is obtained by identifying the term ${ }_{\infty} E\left(E_{\infty}\left(B_{k}\right)\right)$ with ${ }_{0} E\left(E_{0}\left(B_{k+1}\right)\right)$ in the corresponding spectral sequences for $B_{k}, B_{k+1}$, from Theorem 3.8. The remainder of the theorem follows from Theorem 3.9 together with Theorem 4.1 .

Applying Theorem 4.6, together with the results of $\S 2$, we get:

THEOREM 4.7. Let $X$ be an $H$-space, with $H_{*}(X)$ finitely generated. If $H^{*}\left(X ; Z_{p}\right)$ has a generator of dimension $2 m-1$, then $H^{*}(X ; Q)(Q=$ rationals $)$ has a generator of dimension $2 m p^{q}-1$, for some $q, 0 \leqq q<\infty$. If $H^{*}\left(X ; Z_{p}\right)$ has a generator of dimension $2 m$, then $H^{*}(X ; Q)$ has a generator of dimension $2 m p^{k}-1$ for some $k, 0<k<\infty$.

Proof. This follows easily from Theorem 4.6, the results of $\$ 2$ (e.g., Theorem 2.7), and the fact that $B_{\infty}$ is an exterior algebra on odd dimensional generators (see [7, Corollary 4.13]). This last fact shows that $k>0$, since the generator in dimension $2 m$ must bound in the biprimitive spectral sequence, and we get a nontrivial algebra of type I. 
We consider a special case:

THEOREM 4.8. Let $X$ be an $H$-space with $H_{*}(X)$ finitely generated, and suppose that $H^{*}(X ; Q)=\wedge(z)$. Then, if $p \neq 2$,

$$
H^{*}\left(X ; Z_{p}\right)=\wedge(x) \otimes \bigotimes_{i=1}^{m} Z_{p}\left[y_{i}\right] /\left(y_{i}^{h_{i}}\right), \quad h_{i}=p^{n_{i}},
$$

as an algebra, where $\operatorname{dim} y_{i+1}=h_{i} \operatorname{dim} y_{i}, \operatorname{dim} y_{1}=1+\operatorname{dim} x=2 q$, and $\operatorname{dim} z=2 q p^{\alpha}-1$, where $\alpha=\sum_{i=1}^{m} n_{i}$. If $p=2$, the conclusion is the same except when $\operatorname{dim} x=1$, when we allow the possibility that $y_{1}=x^{2}$. Note that $m=0$ is allowed, i.e., $H^{*}\left(X ; Z_{p}\right)$ may be an exterior algebra $\wedge(x)$.

Proof. Since $H^{*}(X ; Q)=\wedge(z)$, it follows that $H^{*}(X) /$ torsion $=\wedge(z)$ so that $B_{\infty}=\left(H^{*}(X) /\right.$ torsion) $\otimes Z_{p}=\wedge(z)$ (where $\wedge(z)$ means the exterior algebra on one generator over the appropriate ring or field in each case). Hence the biprimitive form of $B_{\infty}$ is the exterior algebra on one generator. Then by Theorem 3.11 (applied successively to each term $B_{k}$ of the Bockstein spectral sequence), $B_{1}=H^{*}\left(X ; Z_{p}\right)$ has only one odd dimensional generator in its biprimitive form. Hence

$$
{ }_{0} E\left(E_{0}\left(B_{1}\right)\right) \cong \wedge(x) \otimes \bigotimes_{i=1}^{k} Z_{p}\left[w_{i}\right] /\left(w_{i}^{p}\right)
$$

If we can prove that $\operatorname{dim} w_{1}=(\operatorname{dim} x)+1$ and $\operatorname{dim} w_{i+1}=p\left(\operatorname{dim} w_{i}\right)$, then the result will follow from Theorem 2.7 .

Since all the $w_{i}$ 's are permanent cycles by Theorem 4.1, if $x$ is also a cycle then the differential is zero in ${ }_{0} E\left(E_{0}\left(B_{1}\right)\right)$, so ${ }_{1} E\left(E_{0}\left(B_{1}\right)\right)={ }_{0} E\left(E_{0}\left(E_{1}\right)\right)$. Similarly ${ }_{0} E\left(E_{0}\left(B_{1}\right)\right)={ }_{1} E\left(E_{0}\left(B_{1}\right)\right)=\cdots={ }_{s} E\left(E_{r}\left(B_{l}\right)\right.$ where finally ${ }_{s} d x \neq 0$ in ${ }_{s} E\left(E_{r}\left(B_{l}\right)\right)$. Then ${ }_{s} d x=w_{1}$ (assuming the $w_{i}$ 's are numbered appropriately) and by Theorems 4.6 and 3.10,

$$
{ }_{s+1} E\left(E_{r}\left(B_{l}\right)\right)=H\left({ }_{s} E\left(E_{r}\left(B_{l}\right)\right)\right)=\wedge\left(x_{1}\right) \otimes \bigotimes_{i=2}^{k} Z_{p}\left[w_{i}\right] /\left(w_{i}^{p}\right),
$$

where $x_{1}=\left\{x w_{1}^{p-1}\right\}, \operatorname{dim} x_{1}=p\left(\operatorname{dim} w_{1}\right)-1, \operatorname{dim} w_{1}=\operatorname{dim} x+1$. Continuing in a similar fashion, we get that $w_{2}=d x_{1}$ in some term of the spectral sequence, so that $\operatorname{dim} w_{2}=p\left(\operatorname{dim} w_{1}\right)$. We may continue this argument to show that $\operatorname{dim} w_{i+1}=p\left(\operatorname{dim} w_{i}\right)$ for all $i$, since each $w_{j}$ must be a boundary in some term of the spectral sequence, since the limit is an exterior algebra. This completes the proof.

Theorem 4.8 will be the basis of a thorough study of such $H$-spaces which are rational homology spheres, which will appear elsewhere [9].

5. Applications to multiplicative fibre maps. In this section we study the spectral sequence $\mathscr{E}_{r}$ of a multiplicative fibre map of one $H$-space to another. J. C. Moore [17] has pointed out that over a field of coefficients, this spectral 
sequence is a spectral sequence of Hopf algebras. Using the techniques developed in $\S 3$ we derive some theorems about the structure of such spectral sequences, getting transgression theorems which generalize various known results (see $[12$, exp. $7 ; 5])$. These results are then applied to study relations between the homology and cohomology of an $H$-space and its loop space.

Let $E$ and $B$ be $H$-spaces, with multiplications $\mu: E \times E \rightarrow E$ and $\mu^{\prime}: B \times B \rightarrow B$, and let $f: E \rightarrow B$ be a fibre map such that $f \circ \mu=\mu^{\prime} \circ(f \times f)$. We call $f$ a multiplicative fibre $\operatorname{map}\left({ }^{\mathfrak{3}}\right)$.

Clearly the fibre $F=f^{-1}(e)(e=$ unit of $B)$ is an $H$-space, with $\mu$ as its multiplication. Let us always assume that $B$ and $F$ are connected.

Lemma 5.1. $\pi_{1}(B)$ acts trivially on $H_{*}(F ; K)$ for any coefficient group $K$.

Proof. The action of $\pi_{1}(B)$ on $H_{*}(F ; K)$ may be described as follows:

Let $\phi: I \rightarrow B, \phi(0)=\phi(1)=e$, represent an element $\alpha \in \pi_{1}(B)$. Consider $\phi$ as a homotopy of the constant map of $F \rightarrow B$. Then covering the homotopy $\phi$, we can find a homotopy $\Phi$ of the identity map of $F \rightarrow F \subset E, \Phi: F \times I \rightarrow E$, with $f \circ \Phi=\phi$ (so that for any value of $t, \Phi(F \times t) \subset f^{-1}(\phi(t))$ ). Then $k: F \rightarrow F$ is defined by $k(x)=\Phi(x, 1)$, and its image is in $F$ since $f \circ \Phi(x, 1)=\phi(1)=e$. Then $k_{*}: H_{*}(F ; K) \rightarrow H_{*}(F ; K)$ is the automorphism induced by $\alpha \in \pi_{1}(B)$, and it can be shown to be independent of the choice of $\phi$ representing $\alpha$, or of $\Phi$ covering $\phi$.

We shall show that $\Phi$ may be chosen in such a way that $k$ is homotopic to the identity map, so that $k_{*}=$ identity. We construct $\Phi$ as follows:

Consider $\phi: I \rightarrow B$ as a homotopy of the projection of the map of one point into $F$, namely the inclusion of the unit $e$ of $E$. Let $h: I \rightarrow E$ be a lifting of this homotopy, so that $h(0)=e, f(h(t))=\phi(t)$. Define $\Phi: F \times I \rightarrow E$, by $\Phi(x, t)$ $=\mu(x, h(t))$. Then $\Phi$ is continuous and

$$
\begin{aligned}
f(\Phi(x, t)) & =f(\mu(x, h(t))) \\
& =\mu^{\prime}(f(x), f(h(t)))=\mu^{\prime}(e, \phi(t))=\phi(t)
\end{aligned}
$$

since $x \in F=f^{-1}(e)$. Then $k(x)=\Phi(x, 1)=\mu(x, y)$ where $y=h(1)$. Since $F$ is connected, there is path $g: I \rightarrow F$ with $g(0)=y, g(1)=e$. Then $H(x, s)=\mu(x, g(s))$ defines a homotopy between $k$ and the identity, i.e., $H(x, 0)=k(x), H(x, 1)=x$. Hence $k_{*}=1$ and the lemma is proved.

Since the diagram

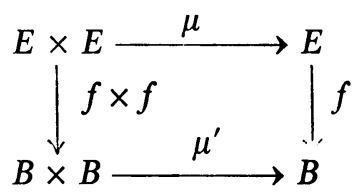

(3) It is easily shown that if $f$ is a homotopy multiplicative fibre map (i.e., $f \circ \mu \sim \mu^{\prime} \circ(f \times f)$ ), then $\mu \sim \bar{\mu}$, such that $f$ is a multiplicative fibre map when $E$ is equipped with the multiplication $\bar{\mu}$. In fact if $f: E \rightarrow B$ is a homotopy multiplicative map, then there is a multiplicative fibre map $f^{\prime}: E^{\prime} \rightarrow B^{\prime}$, with $E \cong E^{\prime}, B \cong B^{\prime}$, and $f^{\prime}$ equivalent to $f$ (in an obvious sense). 
is commutative and $f \times f$ is a fibre map, $\mu$ and $\mu^{\prime}$ induce maps of spectral sequences in homology and cohomology. Let us assume that $H_{i}(B), H_{j}(F)$ are finitely generated for all $i, j$.

Let $K$ be a field, $\mathscr{E}_{r}$ the cohomology spectral sequence (over $K$ ) of the fibering $f$. Then it follows from the results of [13] (cf. [17]) that $\mathscr{E}_{r}(f \times f)=\mathscr{E}_{r}(f)$ $\otimes \mathscr{E}_{r}(f)$ (with the tensor product differential). Hence $\mu$ together with the diagonal map induces a Hopf algebra structure in the spectral sequences associated with $f$. In particular $\mathscr{E}_{2}(f) \cong H^{*}(F ; K) \otimes H^{*}(B ; K)$ as Hopf algebras.

In general, since the product map and the diagonal map preserve both filtration and total degree it follows that $\mathscr{E}_{r}$ is a bigraded Hopf algebra. As usual we will use the filtration degree $p$ and the complementary degree $q$ as our gradations (complementary degree $=$ total degree - filtration degree). Then

$$
d_{r}: \mathscr{E}_{r}^{p, q} \rightarrow \mathscr{E}_{r}^{p+r, q-r+1} .
$$

Let us now consider such bigraded differential Hopf algebras.

Let $A$ be a bigraded differential Hopf algebra over $Z_{p}$. If

$$
d: A^{p, q} \rightarrow A^{p+r, q-r+1}
$$

then we shall say $d$ has bidegree $(r, 1)$.

Lemma 5.2. If $A$ is associative and $d$ has bidegree $(r, 1)$ then $E_{s}(A)$ is a spectral sequence of bigraded Hopf algebras, and $d_{s}$ has bidegree $(r, 1)$.

Lemma 5.3. If $A$ is coassociative and $d$ has bidegree $(r, 1)$ then ${ }_{s} E(A)$ is a spectral sequence of bigraded Hopf algebras, and ${ }_{s} d$ has bidegree $(r, 1)$.

Lemma 5.4. Let $A$ be a bigraded associative, commutative Hopf algebra. Then $E_{0}(A) \cong A$ as bigraded algebras.

Lemma 5.5. Let $A$ and $B$ be biprimitive, bigraded Hopf algebras. Then $A$ and $B$ are isomorphic bigraded Hopf algebras if and only if $\operatorname{rank}\left(A^{p, q}\right)$ $=\operatorname{rank}\left(B^{p, q}\right)$ for all $p$ and $q$.

The proofs of these lemmas are routine extensions of similar proofs about graded Hopf algebras.

Using Lemmas 5.2, 5.3 and 5.5 we may extend Theorem 3.8 to the bigraded case in an obvious manner:

THEOREM 5.6. Let $A$ be a bigraded differential Hopf algebra with differential d of bidegree $(r, 1)$. Suppose $A$ is either associative or coassociative. Then there exists a spectral sequence of biprimitive, bigraded Hopf algebras with differentials of bidegree $(r, 1)$, the first term of the spectral sequence being the biprimitive form of $A$, and the last being the biprimitive form of $H(A)$.

We omit the proof, referring to the proof of Theorem 3.8. 
Theorems 3.9 and 3.10 describe the homology of biprimitive differential Hopf algebras. We shall not write down the bigraded versions of these theorems, but simply use them, referring to the singly graded versions.

The following technical lemma and its consequence are the main tools we shall need.

LEMMA 5.7. Let $A$ be a bigraded, biprimitive differential Hopf algebra with differential $d$ of type $(r, 1), r>1$. Suppose further that $A=B \otimes C \otimes J$, where $B=\sum_{q=0}^{\infty} A^{0, q}=$ the Hopf subalgebra of $A$ of elements of filtration zero, $C=\sum_{p=0}^{\infty} A^{p, 0}=$ the Hopf subalgebra of $A$ of complementary degree zero, $J=$ an exterior algebra on odd dimensional generators. Then $H(A) \cong B^{\prime}$ $\otimes C^{\prime} \otimes J^{\prime}$, where $B^{\prime}=\sum_{q=0}^{\infty} H^{0, q}(A), C^{\prime}=\sum_{p=0}^{\infty} H^{p, 0}(A)$ and $J^{\prime}$ is an exterior algebra on odd dimensional elements. Further $B^{\prime}$ is generated by the cycles in $P(B), C^{\prime}$ is generated by the cycles in $P(C)$ which do not bound, and the generators of $J^{\prime}$ are the cycles of $P(J)$ which do not bound and generators of the following two types: $\left\{y^{p-1}(d y)\right\}$, where $y \in P(B)$ and $\left\{x(d x)^{p-1}\right\}$ where $d x \in P(C)$.

Proof. First we note that since $d$ is of type $(r, 1), r>1$, it follows that no element of $B$ is a boundary, and that all elements of $C$ are cycles. Further, in an even total degree, all primitive elements are sums of primitive elements of $B$ and $C$, i.e., $P(A)=P(B)+P(C)$, in even total degrees. Then the lemma follows from Theorems 3.9 and 3.10 (in their bigraded interpretation).

Now we are in a position to prove the main theorem describing the spectral sequence of a multiplicative fibre map.

THEOREM 5.8. Let $\mathscr{E}_{r}$ be the spectral sequence over $Z_{p}$ of a multiplicative fibre map. Then $\mathscr{E}_{r} \cong B_{r} \otimes C_{r} \otimes M_{r} \otimes N_{r}, r \geqq 2$, as algebras, where $B_{r}=\mathscr{E}_{r}^{0, *}$, $C_{r}=\mathscr{E}_{r}^{*, 0}\left(B_{r}\right.$ and $C_{r}$ are sub-Hopf algebras of $\left.\mathscr{E}_{r}\right) M_{r}=\wedge\left(x_{1}, \cdots, x_{k}\right)$, $N_{r}=\wedge\left(w_{1}, \cdots, w_{l}\right)$, where filtration degree of $x_{i}<r$ for $1 \leqq i \leqq k$, and complementary degree $w_{j}<r-1$, for $1 \leqq j \leqq l$. Further, for each $i, 1 \leqq i \leqq k$, $\operatorname{dim} x_{i}=p^{q}\left(2 m_{i}\right)+1$, where $2 m_{i}=$ the dimension of some generator of $H^{*}\left(F ; Z_{p}\right)$ $=\mathscr{E}_{2}^{0, *}$, and for each $j, 1 \leqq j \leqq l, \operatorname{dim} w_{j}=p^{t_{j}}\left(2 n_{j}\right)-1$, where $2 n_{j}=$ the dimension of some generator of $H^{*}\left(B ; Z_{p}\right)=\mathscr{E}_{2}^{*, 0}$.

Proof. Since $\mathscr{E}_{2}=H^{*}(F ; K) \otimes H^{*}(B ; K)$ the theorem is true for $r=2$, with $M_{2}=N_{2}=Z_{p}$. We proceed by induction, assuming the theorem true for $\mathscr{E}_{r}$.

For any Hopf algebra $L$, denote by ${ }^{0} L$ its biprimitive form. It is obvious that ${ }^{0}\left(L \otimes L^{\prime}\right) \cong{ }^{0} L \otimes{ }^{0} L^{\prime}$. Then ${ }^{0} \mathscr{E}_{r}={ }^{0} B_{r} \otimes{ }^{0} C_{r} \otimes{ }^{0} M_{r} \otimes{ }^{0} N_{r}$.

Since $\mathscr{E}_{r}$ is associative we may apply Theorem 5.6, so that there is a spectral sequence of biprimitive, bigraded, Hopf algebras, whose first term is ${ }^{0} \mathscr{E}_{r}$ and whose last term is ${ }^{0} \mathscr{E}_{r+1}$. Now ${ }^{0} \mathscr{E}_{r}$ satisfies the hypothesis of Lemma 5.7 with $B={ }^{0} B_{r}, C={ }^{0} C_{r}$, and $J={ }^{0} M_{r} \otimes{ }^{0} N_{r}$. By Lemma 5.7, the homology of such 
a Hopf algebra has the same form, so that every term of the spectral sequence satisfies the hypothesis of Lemma 5.7.

Then repeated use of Lemma 5.7 on each term of the spectral sequence of biprimitive Hopf algebras yields the result that ${ }^{0} \mathscr{E}_{r+1}={ }^{0} B_{r+1} \otimes{ }^{0} C_{r+1} \otimes J^{\prime \prime}$, where $J^{\prime \prime}$ is an exterior algebra on odd dimensional generators, ${ }^{0} B_{r+1}={ }^{0} \mathscr{E}_{r+1}^{0, *}$, ${ }^{0} C_{r+1}={ }^{0} \mathscr{E}_{r+1}^{*, 0}$. The way in which generators of $J^{\prime \prime}$ arise is described in Lemma 5.7. In particular we get that generators of the form $\left\{y^{p-1}(d y)\right\}(y \in P(B))$ have filtration degree $r$ since $y$ has filtration degree 0 and $d$ increases filtration by $r$. Similarly generators of the form $\left\{x(d x)^{p-1}\right\} \quad(d x \in P(C))$ have complementary degree $=r-1$, since $d x$ has complementary degree 0 , and $d$ reduces complementary degree by $r-1$. Hence we get these two new types of generators in ${ }^{0} \mathscr{E}_{r+1}$, the other generators coming from generators of $0 \mathscr{E}_{r}$. Note that primitive elements in ${ }^{0} \mathscr{E}_{r}^{0, *}$ and ${ }^{0} \mathscr{E}_{r}^{*, 0}$ have the same dimension as the generators of $\mathscr{E}_{r}^{0, *}, \mathscr{E}_{r}^{*, 0}$, or $p$ th powers of generators by Theorem 2.7. Thus if we put generators of the form $\left\{y_{p-1}(d y)\right\}$ in ${ }^{0} M_{r+1},\left\{x(d x)^{p-1}\right\}$ in ${ }^{0} N_{r+1}$, it follows that ${ }^{0} \mathscr{E}_{r+1}={ }^{0} B_{r+1} \otimes{ }^{0} C_{r+1}$ $\otimes{ }^{0} M_{r+1} \otimes{ }^{0} N_{r+1}$. The result for $\mathscr{E}_{r+1}$ now follows from Theorem 2.7 (in its bigraded version).

If $p \neq 2$, the fact that for the odd dimensional generators of $M_{r+1}, N_{r+1}, x_{i}, w_{j}$, we have $x_{i}^{2}=w_{j}^{2}=0$, follows immediately. When $p=2$, if some $x_{i}^{2}$ or $w_{j}^{2}$ were $\neq 0$, then by Theorem 2.7, we would get an even dimensional generator in ${ }^{0} \mathscr{E}_{r+1}^{p, q}$ with $p \neq 0$ and $q \neq 0$, which is not the case. Hence $x_{i}^{2}=w_{j}^{2}=0$, and these elements generate exterior algebras $M_{r+1}, N_{r+1}$ in $\mathscr{E}_{r+1}$, which concludes the proof.

From Theorem 5.8 we may deduce a number of corollaries.

COROLlaRY 5.9. The primitive elements of $\mathscr{E}$ (the homology spectral sequence of a multiplicative fibre map) are of four types:

(i) Those of filtration zero (i.e., in $\mathscr{E}_{0, *}^{r}$ ).

(ii) Those of complementary degree zero (i.e., in $\left.\mathscr{E}_{*, 0}\right)$.

(iii) Elements of filtration $<r$, of dimension $p^{q}(2 m)+1$ for some $q \geqq 1$, where $2 m=$ dimension of a generator of $H^{*}\left(F ; Z_{p}\right)$.

(iv) Elements of complementary degree $<r-1$, of dimension $p^{t}(2 n)-1$, for some $t \geqq 1$, where $2 n=$ dimension of a generator of $H^{*}\left(B ; Z_{p}\right)$.

Proof. $P(\mathscr{E})=\left(Q\left(\mathscr{E}_{r}\right)\right)^{*}$, where $Q\left(\mathscr{E}_{r}\right)=$ the indecomposable elements of $\mathscr{E}_{r}=F^{1}\left(\mathscr{E}_{r}\right) / F^{2}\left(\mathscr{E}_{r}\right)$. From Theorem 5.8 we see that the indecomposable elements of $\mathscr{E}_{r}$ have filtrations, dimensions, etc., of the four types listed.

COROLlary 5.10. The primitive elements of $\mathscr{E}_{r}$ are of the four types, as in Corollary 5.9.

Proof. According to [16, Proposition 4.23] any primitive element in an associative, commutative Hopf algebra over $Z_{p}$ which is decomposable is a $p$ th 
power. By Theorem 5.8, the indecomposable elements of $\mathscr{E}_{r}$ have the four types listed. But if $\zeta: \mathscr{E}_{r} \rightarrow \mathscr{E}_{r}$ is defined by $\zeta(x)=x^{p}$, then $\zeta\left(\mathscr{E}_{r}\right)=\zeta\left(B_{r}\right) \otimes \zeta\left(C_{r}\right)$ (for $p$ th powers are zero in an exterior algebra, e.g., $\left.M_{r}, N_{r}\right)$. Hence the primitive elements of $\mathscr{E}_{r}$ which are decomposable are either of type (i) or (ii), which completes the proof of the corollary.

COROLlaRY 5.11. If $x \in P\left(\mathscr{E}_{r}^{h, k}\right)$ and if either $h \neq 0$, or $h=0$ and $k \neq p^{t}(2 n)-2$, for any $t \geqq 1$, for $2 n=$ dimension of any generator of $H^{*}\left(B ; Z_{p}\right)$, then $x$ is transgressive.

COROLlaRY 5.12. If $y \in P\left(\mathscr{E}_{l, s}\right)$ and if either $s \neq 0$ or $s=0$ and $l \neq p^{q}(2 m)+2$ for any $q \geqq 1$, and for $2 m=$ dimension of any generator of $H^{*}\left(F ; Z_{p}\right)$, then $y$ is transgressive.

Recall that an element $x \in \mathscr{E}_{r}, k$ is called transgressive if $d_{j} x=0$ for $j<k-1$, i.e., if all $d_{j}$ are zero on $x$ except possibly for $d_{k-1}$ which sends $x$ to $\mathscr{E}_{k-1}^{*, 0}$. If $x$ is primitive, then $d_{j} x$ is primitive. Of the four types of primitive elements in Corollary 5.9, types (i) and (iii) cannot be boundaries. Hence $d_{j} x$ is either of type (ii) or (iv). If $d_{j} x$ is of type (iv) then $\operatorname{dim} x=p^{t}(2 n)-2$. Otherwise $d_{j} x$ has complementary degree zero (type (ii)) and $x$ is transgressive, and Corollary 5.11 follows. Corollary 5.12 follows by a similar argument utilizing Corollary 5.9.

We now restrict our hypothesis to a special case of great interest.

Let $X$ be a connected $H$-space, $E$ the space of paths of $X$ starting from the identity, $f: E \rightarrow X$, the projection. Then $f$ is a multiplicative fibre map, and the fibre $f^{-1}(e)=\Omega X$, the loop space of $X$. Let us suppose further that $X$ is simply connected, so that $\Omega X$ is arcwise connected. Let $\mathscr{E}_{r}$ (resp. $\mathscr{E}$ ) be the spectral sequence of $f$ in cohomology (resp. homology) $\bmod p$, where $p$ is prime as usual.

Since $E$ is contractible we may define the suspension maps

$$
\begin{aligned}
& \sigma^{*}: H^{i}(X ; G) \rightarrow H^{i-1}(\Omega ; G), \text { for } i>1, \\
& \sigma_{*}: H_{i}(\Omega ; G) \rightarrow H_{i+1}(X ; G), \text { for } i \geqq 1,
\end{aligned}
$$

as follows: $\sigma^{*}=\delta^{*-1} f^{*} j^{*-1}, \sigma_{*}=j_{*}^{-1} f_{*} \partial_{*}^{-1}$ where $j: X \rightarrow(X, e)$, and $\delta^{*}: H^{i-1}(\Omega ; G) \rightarrow H^{i}(E, \Omega ; G)$ and $\partial_{*}: H_{i+1}(E, \Omega ; G) \rightarrow H_{i}(\Omega ; G)$ are the boundary operators associated with the pair $(E, \Omega)$. The following are well known properties of the suspensions:

(1) $\sigma^{*}$ and $\sigma_{*}$ annihilate decomposable elements.

(2) $\sigma^{*}$ and $\sigma_{*}$ are dual maps if $G$ is a field.

(3) $x=\sigma^{*} y$ if and only if $x$ is transgressive (its "transgression image" is $y$ ). Similarly for $\sigma_{*}$.

Let, as usual, $P(A)$ be the primitive elements, $Q(A)=\bar{A} / \bar{A} \bar{A}, A$ a Hopf algebra. Then $\sigma^{*}\left(Q\left(H^{*}\left(X ; Z_{p}\right)\right)\right) \subset P\left(H^{*}\left(\Omega X ; Z_{p}\right)\right), \sigma_{*}\left(Q\left(H_{*}\left(\Omega X ; Z_{p}\right)\right)\right) \subset P\left(H_{*}\left(X ; Z_{p}\right)\right)$. Applying Corollaries 5.11 and 5.12 we get: 
THEOREM 5.13. Let $X$ be an arcwise connected, simply connected H-space. Then

$$
\sigma_{*}: Q\left(H_{k}\left(\Omega X ; Z_{p}\right)\right) \rightarrow P\left(H_{k+1}\left(X ; Z_{p}\right)\right)
$$

is mono for $k \neq p^{t}(2 n)-2$, where $2 n=$ dimension of some generator of $H^{*}\left(X ; Z_{p}\right), t \geqq 1$, and $\sigma_{*}$ is epi for $k \neq p^{q}(2 m)+1,2 m=$ dimension of some generator of $H^{*}\left(\Omega X ; Z_{p}\right), q \geqq 1$. In particular, $\sigma_{*}$ is mono if $k \neq \equiv-2 \bmod (2 p)$, epi if $k \neq 1 \bmod (2 p)$.

This result generalizes a result of J. C. Moore [12, exp. 7, Theorem VI, 4]. The proof follows easily from Corollaries 5.11 and 5.12, using the dualities of $\sigma^{*}=\left(\sigma_{*}\right)^{*}$, and $P\left(A^{*}\right)=(Q(A))^{*}$, etc. It is easy to obtain a similar result for cohomology:

THEOREM 5.14. Let $X$ be an arcwise connected, simply connected H-space. Then

$$
\sigma^{*}: Q\left(H^{l}\left(X ; Z_{p}\right)\right) \rightarrow P\left(H^{l-1}\left(\Omega X ; Z_{p}\right)\right)
$$

is mono for $l \neq p^{q}(2 m)+2$, where $2 m=$ dimension of some generator of $H^{*}\left(\Omega X ; Z_{p}\right) \quad q \geqq 1$ and $\sigma^{*}$ is epi for $l \neq p^{t}(2 n)-1,2 n=$ dimension of some generator of $H^{*}\left(X ; Z_{p}\right), t \geqq 1$. In particular $\sigma^{*}$ is mono if $l \neq \equiv 2 \bmod (2 p)$, epi if $l \not \equiv-1 \bmod (2 p)$.

This follows from Theorem 5.13.

Theorem 5.13 was used by Liulevicius [15] to deduce some properties of certain universal examples for cohomology operations.

These theorems may be used to compute homology of loop spaces. As a simple application of Theorem 5.13, we have the following $\left({ }^{4}\right)$ :

THEORIM 5.15. Let $X$ be an arcwise connected, simply connected $H$-space. Suppose $H^{*}(X ; K)=\wedge\left(x_{1}, \cdots, x_{m}, \cdots\right)$, an exterior algebra on generators $x_{1}, x_{2}, \cdots, \operatorname{dim} x_{i}=2 n_{i}+1, K$ a field. Then $H_{*}(\Omega X ; K)=K\left[y_{1}, \cdots, y_{m}, \cdots\right]$, $\operatorname{dim} y_{i}=2 n_{i}, x_{i}\left(\sigma_{*} y_{i}\right) \neq 0$.

Proof. If characteristic of $K=p$, then $H^{*}(X ; K)=H^{*}\left(X ; Z_{p}\right) \otimes K$, and $H_{*}(\Omega X ; K)=H_{*}\left(\Omega X ; Z_{p}\right) \otimes K$. Then it suffices to prove the theorem for $K=Z_{p}$ $\left(Z_{0}=Q\right.$, the rational numbers).

Since $P\left(H_{*}\left(X ; Z_{p}\right)\right)=\left(Q\left(H^{*}\left(X ; Z_{p}\right)\right)\right)^{*}$, it follows that there are elements $\bar{x}_{i} \in P\left(H_{2 n_{i}+1}\left(X ; Z_{l}\right)\right)$ such that $x_{i}\left(\bar{x}_{i}\right) \neq 0$. By Theorem 5.13, there are elements $y_{i} \in H_{2 n_{i}}\left(\Omega X ; Z_{p}\right)$ such that $\bar{x}_{i}=\sigma_{*}\left(y_{i}\right)$, for all $i$. Define a filtered differential Hopf algebra $A$, by $A=K\left[y_{1}, \cdots, y_{m}, \cdots\right] \otimes \wedge\left(\bar{x}_{1}, \cdots, \bar{x}_{m}, \cdots\right)$, with $d \bar{x}_{i}=y_{i}$,

(4) The referee has pointed out that this theorem follows without the hypothesis that $X$ is an $H$-space, by using the spectral sequence of the cobar construction. 
filtered by degree in $\wedge\left(\bar{x}_{1}, \cdots\right), \bar{x}_{i}, y_{i}$, primitive. If $\mathscr{E}^{r r}=$ the spectral sequence of the space of paths of $X(\bmod p)$, we define a map of $E^{r}(A)(r \geqq 2)$ into $\mathscr{E}^{r}$, by induction. $E^{2}(A) \cong A$, so we define $f^{2}\left(y_{i}\right)=y_{i} \in \mathscr{E}^{2}=H_{*}\left(\Omega X ; Z_{p}\right) \otimes H_{*}\left(X ; Z_{p}\right)$, $f^{2}\left(\bar{x}_{i}\right)=\bar{x}_{i} \in \mathscr{E}^{2}$. Since $K\left[y_{1}, \cdots\right]$ is a free associative, commutative algebra, we may extend $f^{2}$ to $K\left[y_{1}, \cdots\right]$ into $H_{*}\left(\Omega X ; Z_{p}\right) \subseteq \mathscr{E}^{2}$. Unfortunately, since $H_{*}\left(X ; Z_{p}\right)$ may not be associative, we must exercise more care defining the map on $\wedge\left(\tilde{x}_{1}, \cdots\right)$. We choose a fixed order of multiplication (or parenthesizing in products) in $H_{*}\left(X ; Z_{p}\right)$ and extend the map $f^{2}$, already defined on the generators $\bar{x}_{i}$, to all of $\wedge\left(\bar{x}_{1}, \cdots\right)$ by using the fixed order of multiplication in $H_{*}\left(X ; Z_{p}\right)$. Note that this is a homomorphism of the coalgebra $\wedge\left(\bar{x}_{1}, \cdots\right)$ into the coalgebra $H_{*}\left(X ; Z_{p}\right)$ since the $\bar{x}_{i} \in P\left(H_{*}\left(X ; Z_{p}\right)\right)$. Thus the dual map $f_{2}: H^{*}\left(X ; Z_{p}\right) \rightarrow\left(\wedge\left(\bar{x}_{1}, \cdots\right)\right)^{*}$ is a ring homomorphism, and a set of generators $\left(\bar{x}_{i}\right)^{*} \in$ image $f_{2}$, so $f_{2}$ is onto $\left(\wedge\left(\bar{x}_{1}, \cdots\right)\right)^{*}$. Since $\left(\Lambda\left(\bar{x}_{1}, \cdots\right)\right)^{*}$ and $H^{*}\left(X ; Z_{p}\right)$ have the same rank over $Z_{p}$ in each dimension it follows that $f_{2}$ and hence $f^{2}$ are isomorphisms in complementary degree zero, i.e., $f^{2}: \wedge\left(\bar{x}_{1}, \cdots\right) \rightarrow H_{*}\left(X ; Z_{p}\right)$ is an isomorphism.

Since the $\bar{x} \in H_{*}\left(X ; Z_{p}\right)$ are transgressive, $\bar{x}_{i}=\sigma_{*} y_{i}$, it follows that we may define $f^{r}$ by induction, starting from $f^{2}$, to be a homomorphism of spectral sequences. Further, it is easy to check that $A$ is acyclic, so $f^{\infty}$ is an isomorphism. Hence by the Comparison Theorem for spectral sequences (see [18] or [14, Proposition 5.3]) $f^{2}$ is an isomorphism on $\mathscr{E}_{0, *}^{2}$, i.e., $f^{2}: K\left[y_{1}, \cdots\right] \rightarrow H_{*}\left(\Omega X ; Z_{\rho}\right)$ is an isomorphism, and the theorem is proved.

Theorem 5.14 may be used in conjunction with the results of [10] to compute the homology ring $H_{*}\left(\Omega^{k} s^{k} X ; Z_{p}\right)$ for $k<2 p$ as a certain functor of $H_{*}\left(X ; Z_{p}\right)$, which was the main result of [5].

6. Applications to Lie groups. In this section we apply the results of $\S \S 4$ and 5 to studying homology of Lie groups, or more generally, $H$-spaces whose homology is finitely generated, and whose loop space has no torsion in homology. We prove theorems on which dimension torsion can first occur in the homo-ogy of such an $\mathrm{H}$-space and the relationship of this dimension with the dimension of generators in the rational cohomology. We also show that for such an $\mathrm{H}$ space $X, H_{*}\left(X ; Z_{p}\right)$ is associative and commutative, if and only if $H^{*}\left(X ; Z_{p}\right)$ is primitively generated.

Since the homology of all Lie groups is now known, the results on torsion contain nothing new in this case.

Suppose now that $X$ is an $H$-space, arcwise connected and simply connected, such that $H_{*}(X)$ has no torsion. We recall that it is a theorem of Bott [4] that if $G$ is a Lie group, then $H_{*}(\Omega G)$ has no torsion. If $H^{*}(X ; Q)$ is finitely generated, then by Hopf's Theorem (see [16]) $H^{*}(X ; Q)=\wedge\left(x_{1} \cdots, x_{m}\right)$, so Theorem 5.15 applies, and $H_{*}(\Omega X ; Q)=Q\left[y_{1}, \cdots, y_{m}\right]$ and in particular $H_{q}(\Omega X ; Q)=H^{q}(\Omega X ; Q)$ $=0$ if $q$ is odd. Since $H_{*}(\Omega X)$ is torsion free, $H_{*}(\Omega X) \rightarrow H_{*}(\Omega X ; Q)$ is mono. Hence we get: 
Lemma 6.1. Let $X$ be an $H$-space, connected and simply connected such that $H^{*}(X ; Q)$ is a finite $Q$ module, and $H_{*}(\Omega X)$ has no torsion. Then $H_{q}(\Omega X)=0$ for $q$ odd, so that $H_{q}(\Omega X ; G)=0$ for $q$ odd, or any coefficient group $G$.

This implies that image $\sigma^{*}=0$ in $H_{q}(X ; G)$ for $q$ even. Then we get

THEOREM 6.2. Let $X$ be a connected, simply connected $H$-space, with $H^{*}(X ; Q)$ a finite $Q$ module and $H_{*}(\Omega X)$ torsion free. Then $P\left(H_{2 m}\left(X ; Z_{p}\right)\right)=0$ if $m \neq \equiv$ $(\bmod p)$.

For $P\left(H_{2 m}\left(X ; Z_{p}\right)\right)=$ image $\sigma_{*}$, when $m \neq \equiv 1(\bmod p)$, by Theorem 5.13.

Lemma 6.3. Let $A$ be a differential Hopf algebra over $Z_{p}, A_{0}=Z_{p}$, and suppose $d\left(A_{k}\right)=0$ for $k<m$. Then $d\left(A_{m}\right) \subseteq P(A)$.

The proof is straightforward (see [8, Lemma 8.13]).

Lemma 6.4. Let $X$ be a connected $H$-space, with $H_{i}\left(X ; Z_{p}\right)=0$ for $i>N$, for some $N$. Let $s$ be the smallest integer for which $H^{s}(X)$ has p-torsion. Then $s=2 m$, and if $p \neq 2$, then $H_{s}\left(X ; Z_{p}\right)$ has a primitive element. Further, if $p=2$, and $m$ is even, then $H_{s}\left(X ; Z_{2}\right)$ has a primitive element.

Proof. In the Bockstein spectral sequence $B_{k}$ in cohomology $\bmod p$ of $X$, the lowest dimensional element $x_{k}$ in image $\beta_{k}$ is primitive by Lemma 6.3. The lowest dimensional $x_{k}$ (call it $x$ ) occurs in dimension $s$, i.e., in the dimension where $p$-torsion first occurs in $H^{*}(X)$. Let $x=\beta_{k} y, \quad B_{1}^{q}=B_{2}^{q}=\cdots=B_{k}^{q}$, $q \leqq s$. Since $x$ is primitive, if $s$ is odd, then $x$ is indecomposable, by [16, Proposition 4.23]. But then $s-1=\operatorname{dim} y$ is even, so that $x=\beta_{k} y$ is decomposable, by Lemma 4.5. This is a contradiction, so that $s$ must be even, $s=2 m$.

Now $B_{1}=B_{\infty}$ in dimension $<s-1$, so that all generators of $B_{1}$ of dimension $<s$ are odd dimensional. If $p \neq 2$, then there are no nonzero $p$ th powers in $B_{1}^{s}$, since $p$ th powers of odd dimensional generators are zero. By [16, Proposition 4.23] if $x$ is decomposable, it is a $p$ th power, since $x$ is primitive. Hence $x$ is not decomposable, so $H_{s}\left(X ; Z_{p}\right)$ has a primitive element, dual to $x$.

If $p=2$, and if $x$ is decomposable, then $x=u^{2}$. If $g$ is an odd dimensional generator of $H^{2 n+1}\left(X ; Z_{2}\right)$, then $g^{2}=S q^{2 n+1} g=S q^{1} S q^{2 n} g \in$ image $\beta_{1}\left(\beta_{1}=S q^{1}\right)$. Hence, squares of odd dimensional generators $g$, are zero if $\operatorname{dim} g<m$, since $g^{2}$ is a boundary and there are no boundaries in dimensions $<s=2 m$. If $x=u^{2}$, therefore, $u$ must be a generator, hence odd dimensional, and $m$ is odd. If $m$ is even, then $x$ is indecomposable and $H_{s}\left(X ; Z_{2}\right)$ has a primitive element, dual to $x$. This completes the proof of the lemma. (Note that by the above argument, if $P\left(H_{s}\left(X ; Z_{2}\right)\right)=0$, then the 2-torsion in $H^{s}(X)$ is of order 2.)

LEMMA 6.5. Let $X$ be as in Lemma 6.4, and let $t=$ the smallest integer such that $H^{t}(X)$ has higher p-torsion, i.e., elements of order $p^{2}$. Then $t$ is even, and $H_{t}\left(X ; Z_{p}\right)$ has a primitive element $\xi,\{\xi\}=\xi^{\prime} \neq 0$ in $B_{r}, \beta_{r} \xi^{\prime} \neq 0$, for some $r>1$. 
Proof. By an argument identical with that in the proof of Lemma 6.4, we may conclude that if $x$ is a lowest dimensional element in the image $\beta_{r}, r>1$, in $B_{r}$, then $x$ is indecomposable in $B_{2}^{t}$ and $t$ is even. Note that $B_{2}^{q}=B_{3}^{q}=\cdots=B_{r}^{q}$, for $q \leqq t$, if we suppose $r$ is the smallest integer $>1$, such that image $\beta_{r} \neq 0$ in $B_{r}^{t}$, and $t$ is smallest integer such that some image $\beta_{k} \neq 0$ in $B_{k}^{t}, k>1$.

If we consider the spectral sequence $E_{q}\left(B_{1}\right)$ in dimensions $<t$, we find that $E_{\infty}\left(B_{1}\right) \cong B_{2}$ as algebras, in these dimensions, since both are exterior algebras on odd dimensional generators in dimensions $<t$, and the ranks are the same in dimensions $\leqq t$. Further $E_{0}\left(B_{1}\right) \cong B_{1}$ as algebras. Since $E_{\infty}\left(B_{1}\right)$ is an exterior algebra in dimensions $<t$, jt follows that each even dimensional primitive element of dimension $<t$ of $E_{0}\left(B_{1}\right) !$ must bound in the spectral sequence. Since generators are in filtration $1, d_{q}$ increases filtration by $q$, and $d_{q}$ is zero on elements of filtration zero, it follows that eachisuch even dimensional generator must bound in $E_{0}\left(B_{1}\right)$. Then $E_{1}\left(B_{1}\right)$ is generated by odd dimensional elements so that if $p \neq 2, E_{1}\left(B_{1}\right)=E_{\infty}\left(B_{1}\right) \cong B_{2}$ in dimensions $\leqq t$.

We now show that if $x$ is indecomposable in $\left(E_{\infty}\left(B_{1}\right)\right)^{t}$, then there is an indecomposable element $x^{\prime}$ in $E_{0}\left(B_{1}\right),\left\{x^{\prime}\right\}=x \in E_{\infty}\left(B_{1}\right)$. Consider the Hopf subalgebra of $E_{0}\left(B_{1}\right)$ generated be elements of dimension $<t$, and their boundaries. This is a differential Hopf subalgebra of $E_{0}\left(B_{1}\right)$; every even dimensional generator is primitive and a boundary. Its homology is generated by odd dimensional primitive generators. Similarly, the Hopf subalgebra $C$ of $E_{r}\left(B_{1}\right)$ generated by elements of dimension $<t$ and their boundaries, is generated by odd dimensional primitive generators for $r>0$, and the homology $H(C)$ also has this property. Hence, an indecomposable element $x \in E_{r+1}\left(B_{1}\right)$ indimension $t$ cannot be in the image of $H(C)$, so that if $x=\left\{x^{\prime}\right\}, x^{\prime} \in E_{r}\left(B_{1}\right)$, then $x^{\prime}$ is indecomposable.

Hence if $x$ is a lowest dimensional element in image $\beta_{r}(r>1)$ in $B_{r}^{t}$, then for any $x^{\prime} \in B_{1}^{t}$ with $\left\{x^{\prime}\right\}=x$ in $B_{2}^{t}, x^{\prime}$ is indecomposable in $B_{1}=H^{*}\left(X ; Z_{p}\right)$. Hence since there is an element in $B_{1}^{t}$, which is not a boundary modulo decomposable elements, it follows that there is a primitive element in $H_{t}\left(X ; Z_{p}\right)$, which is a cycle under $\beta^{1}$, and has a nonzero boundary in $B_{r}$. This completes the proof of Lemma 6.5.

We may now apply Lemmas 6.4 and 6.5 and Theorem 6.2, to get:

THEOREM 6.6. Let $X$ be a connected, simply connected $H$-space with $H_{i}\left(X ; Z_{p}\right)=0$ for $i>N$, for some $N$, and suppose $H_{*}(\Omega X)$ has no torsion. Then if $H^{s}(X)$ has p-torsion, and $H^{i}(X)$ has no p-torsion for $i<s$, then $s=2 m$ and $m \equiv 1 \bmod p$. Similarly, if $t$ is the lowest dimension for which $H^{t}(X)$ has higher $p$-torsion $\left(p^{2}\right.$-torsion), then $t=2 q$, and $q \equiv 1 \bmod p$.

Proof. $H_{i}\left(X ; Z_{p}\right)=0$ for $i>N$ implies that $H_{i}(X ; Q)=0$ for $i>N$. Hence Theorem 6.2 applies, and $P\left(H_{2 m}\left(X ; Z_{p}\right)\right)=0$ if $m \neq \equiv 1 \bmod p$. But by Lemmas 6.4 and 6.5, $P\left(H_{2 m}\left(X ; Z_{p}\right)\right) \neq 0$, and $P\left(H_{2 q}\left(X ; Z_{p}\right)\right) \neq 0$, unless $p=2$ and $m$ is odd. 
But in case $p=2, m \equiv 1 \bmod 2$ is exactly the statement that $m$ is odd. q.e.d. If $P\left(H_{2 m}\left(X ; Z_{p}\right)\right) \neq 0$, then $Q\left(H^{2 m}\left(X ; Z_{p}\right)\right) \neq 0$, or in other words, $H^{*}\left(X ; Z_{p}\right)$ has a generator of dimension $2 \mathrm{~m}$. Then we may apply Theorem 4.7 to the situation of Theorem 6.6, and we get:

THEOREM 6.7. Let $X$ be a connected, simply connected $H$-space, with $H_{i}\left(X ; Z_{p}\right)$ $=0$ for $i>N$, for some $N$, and suppose $H_{*}(\Omega X)$ has no torsion. If $H^{*}(X)$ has p-torsion, then $H^{*}(X ; Q)$ has a generator of dimension $2 m p^{k}-1$ for some $k \geqq 1$, where $m \equiv 1 \bmod p$, and $2 m$ is the lowest dimension where $H^{*}(X)$ has p-torsion. If $H^{*}(X)$ has higher p-torsion, then $H^{*}(X ; Q)$ has a generator of dimension $2 q p^{l}-1$ for some $l \geqq 1$, where $q \equiv 1 \bmod p$, and $2 q$ is the lowest dimension where $H^{*}(X)$ has higher p-torsion.

REMARKS. (1) It will be shown in [9] that in Theorem $6.7, l \geqq 2$.

(2) Theorem 6.7 can be extended to the nonsimply connected case by using the results of [6].

Now we turn to the problem of commutativity of $H_{*}\left(X ; Z_{p}\right)$.

THEOREM 6.8. Let $X$ be a connected, simply connected $H$-space with $H_{i}\left(X ; Z_{p}\right)$ $=0$ for $i>N$, for some $N$, and $H_{*}(\Omega X)$ torsion free. Then $H_{*}\left(X ; Z_{p}\right)$ is associative and commutative if and only if $H^{*}\left(X ; Z_{p}\right)$ is primitively generated $\left(H^{*}\left(X ; Z_{p}\right)\right.$ is primitive).

Proof. By [16, Theorem 4.9] (Theorem 2.1), $H^{*}\left(X ; Z_{p}\right)$ is primitive if and only if $H_{*}\left(X ; Z_{p}\right)$ is associative, commutative and has all $p$ th powers zero, i.e., $u^{p}=0$ forlany $u \in H_{q}\left(X ; Z_{p}\right), q>0$. It remains only to show then, that if $H_{*}\left(X ; Z_{p}\right)$ is associative and commutative, then $u^{p}=0$ for any $u \in H_{q}\left(X ; Z_{p}\right)$. The map $\zeta: H_{*}\left(X ; Z_{p}\right) \rightarrow H_{*}\left(X ; Z_{p}\right)$ given by $\zeta(x)=x^{p}$ is a map of Hopf algebras if $H_{*}\left(X ; Z_{p}\right)$ is associative and commutative. Hence the lowest dimensional element $u^{p}$ in image $\zeta$ is primitive, and if $p \neq 2$ its dimension is divisible by $2 p$ (since $u$ must be even dimensional in order that $\left.u^{2} \neq 0\right)$. But by Theorem $6.2, P\left(H_{2 m}\left(X ; Z_{p}\right)\right)=0$ unless $m \equiv 1 \bmod p$, so that if $p \neq 2$ image $\zeta=0$ and $u^{p}=0$ for all $u \in H_{q}\left(X ; Z_{p}\right)$, $q>0$.

If $p=2$, a similar argument shows that the lowest dimensional $u \in H_{q}\left(X ; Z_{2}\right)$ such that $u^{2} \neq 0$ occurs in an odd dimension $q$. If $u$ were decomposable then $u^{2}=0$. Hence $u$ is indecomposable. But, since $H^{*}\left(X ; Z_{2}\right)$ is biassociative and bicommutative, we have that $P\left(H_{q}\left(X ; Z_{2}\right)\right) \cong Q\left(H_{q}\left(X ; Z_{2}\right)\right)$ for $q$ odd, i.e., the odd dimensional generators of $H_{*}\left(X ; Z_{2}\right)$ may be chosen primitive. Hence there exists $u^{\prime} \in P\left(H_{q}\left(X, Z_{2}\right)\right)$, with $u^{\prime 2} \neq 0$. By Theorem $5.13, u^{\prime}=\sigma_{*} y$. Then by $[14$, Theorem 5.1] or [10; Theorem 2] $u^{\prime 2}=\sigma_{*}\left(Q_{1}(y)\right)$, and $Q_{1}(y) \in H_{2 q-1}\left(\Omega X ; Z_{2}\right)$. But $H_{2 q-1}\left(\Omega X ; Z_{2}\right)=0$ by Lemma 6.1 , so that $u^{\prime 2}=0$, and $u^{2}=0$ for any $u \in H_{q}\left(X ; Z_{2}\right)$, for $q>0$. This completes the proof of the theorem.

REMARK. This theorem may also be extended to the nonsimply connected case, if $p \neq 2$ using results of [6]. We assume that if $\tilde{X}$ is the universal covering 
space of $X$, that $H^{*}(\Omega \tilde{X})$ has no torsion. Then, by the results of [6], if $H^{*}\left(X ; Z_{p}\right)$ has a generator of dimension $>2, H^{*}\left(\tilde{X} ; Z_{p}\right)$ has a generator of the same dimension. Thus, if $p \neq 2$, and some $u^{p} \neq 0$ in $H_{*}\left(X ; Z_{p}\right)$, then $H_{*}\left(X ; Z_{p}\right)$ has a primitive element of dimension $2 m p, m \geqq 1$, so $H^{2 m p}\left(X ; Z_{p}\right)$ contains a generator. Then $H^{2 m p}\left(\tilde{X} ; Z_{p}\right)$ also contains a generator, so that $P\left(H_{2 m p}\left(\tilde{X} ; Z_{p}\right)\right) \neq 0$ and the remainder of the proof is the same.

7. Appendix. In this section we sketch the proof of Theorem 2.1 [16, Theorem 4.9], for completeness. The proof presented is that of [16] with modified notation.

Let $A$ be a Hopf algebra over $Z_{p}$ with commutative, associative multiplication, and such that $x^{p}=0$ for $x \in \bar{A}$. Let $y \in P(A)$. We wish to show that $y$ is indecomposable. The proof proceeds by induction on the number of generators of $A$. Since for any fixed $m, A_{m}$ is contained in some finitely generated Hopf subalgebra of $A$, if we prove Theorem 2.1 for all finitely generated Hopf algebras, it follows for all Hopf algebras.

Let $A$ have $n$ generators, let $B$ be the Hopf subalgebra of $A$ generated by the first $n-1$ generators (ordered by increasing dimension). Let $C=A / \bar{B} A(C=A / / B$ in the notation of [16]). Then $C$ is a Hopf algebra (becalise $A$ is associative and commutative and $B$ is a Hopf subalgebra), the map $f: A \rightarrow C$ is a homomorphism of Hopf algebras, and $C=Z_{p}[z] /\left(z^{p}\right)$ where $z=f(\gamma), \gamma$ being the $n$th generator of $A$. The element $z$ generates $P(C)$. Hence $f(y)=\lambda z, \lambda \in Z_{p}$. If $\lambda \neq 0$, then $f(y)$ is indecomposable, so $y$ is indecomposable and we are done. If $\lambda=0$, then $y \in$ kernel $f=\bar{B} A$, i.e., $y=\sum_{i=0}^{q} b_{i} \gamma^{i}, b_{i} \in \bar{B}$, where $\gamma^{0}=1$. We shall show that $q=0$, i.e., that $y \in B$, so that $y \in P(B)$, so by the induction hypothesis, $y$ is indecomposable in $B$, i.e., $y$ is a generator of $B$. But the generators of $B$ are a subset of the generators of $A$, so that $y$ is indecomposable in $A$. It remains to show that $q=0$.

Clearly $\psi \gamma=\gamma \otimes 1+1 \otimes \gamma+Q$, where $Q \in \bar{B} \otimes \vec{B}$. Let us take the element $\psi y \in A \otimes A$, and apply $1 \otimes f: A \otimes A \rightarrow A \otimes C$. Finally project $A \otimes C$, onto $A \otimes C_{m q}$ where $\gamma \in A_{m}$. Then $y$ goes to $b_{q} \otimes \gamma^{q}$ in $A \otimes C_{m q}$. But $y$ primitive in $A$ implies that image $y$ in $A \otimes C_{m q}$ is $1 \otimes f(y)=0$, so that $b_{q}=0$, and by induction down on $q, y \in B$ and we are done. This proves Theorem 2.1 in one direction.

Suppose the primitive elements of $A$ are indecomposable. Define $[a, b]=a b$ $-(-1)^{\alpha \beta} b a$, for $\mathrm{a} \in A_{\alpha}, b \in A_{\beta}$. One can show that the lowest dimensional eleinent of the form $[a, b] \neq 0$ is primitive. Since primitive elements are indecomposable, it follows that $[a, b]=0$ for all $a, b \in A$, and $A$ is commutative. A similar argument using the operation $\langle a, b, c\rangle=(a b) c-a(b c)$, shows that $A$ is associative. The map $\xi: A \rightarrow A$ defined by $\xi(x)=x^{p}$, is a map of Hopf algebras over $Z_{p}$ if $A$ is associative and commutative. Hence the lowest dimensional nonzero element $x^{p}$ in image $\xi$ is primitive and decomposable. It follows that $x^{p}=0$ for all $x \in \bar{A}$ and Theorem 2.1 is proved.

Note that the proof of Theorem 2.1 for rational coefficients is the same, except that the condition $x^{p}=0$ no longer plays a role. 


\section{BIBLIOGRAPHY}

1. S. Araki, Differential Hopf algebras and the cohomology mod 3 of the compact exceptional groups $E_{7}$ and $E_{8}$, Ann. of Math. (2) 73 (1961), 404-435.

2. A. Borel, Sur la cohomologie des espaces fibres principaux et des espaces homogènes de groupes de Lie compacts, Ann. of Math. (2) 57 (1953), 115-207.

3. - Sous-groupes commutatifs et torsion des groupes de Lie compact connexes, Tôhoku Math. J. (2) 13 (1961), 216-240.

4. R. Bott, An application of the Morse theory to the topology of Lie groups, Bull. Soc. Math. France 84 (1956), 251-282.

5. W. Browder, Homology of loop spaces, Thesis, Princeton Univ., Princeton, N. J., 1958.

6. - The cohomology of covering spaces of H-spaces, Bull. Amer. Math. Soc. 65 (1959), 140-141.

7. — Torsion in H-spaces, Ann. of Math. (2) 74 (1961), 24-51.

8. - Homotopy commutative H-spaces, Ann. of Math. (2) 75 (1962), 283-311.

9. - Higher torsion in H-spaces, Trans. Amer. Math. Soc. (to appear).

10. - Homology operations and loop spaces, Illinois J. Math. 4 (1960), 347-357.

11. H. Cartan and S. Eilenberg, Homological algebra, Princeton Univ. Press, Princeton, N. J., 1956.

12. H. Cartan and J. C. Moore, Seminaire Ecole Normale Supérieure, Paris, 1959-60.

13. V. K. A. M. Guggenheim and J. C. Moore, Acyclic models and fibre spaces, Trans. Amer. Math. Soc. 85 (1957), 265-306.

14. T. Kudo and S. Araki, Topology of $H_{n}$-spaces and $H$-squaring operations, Mem. Fac. Sci. Kyushu Univ. Ser. A 10 (1956), 85-120.

15. A. Liulevicius, The factorization of cyclic reduced powers by secondary cohomology operations, Proc. Nat. Acad. Sci. U.S.A. 46 (1960), 978-981.

16. J. Milnor and J. C. Moore, On the structure of Hopf algebras, Princeton Univ. Princeton, N. J., 1959 (mimeographed), (to appear).

17. J. C. Moore, The double suspension and p-primary components of the homotopy groups of spheres, Bol. Soc. Mat. Mexicana 1 (1956), 28-37.

18. E. C. Zeeman, A proof of the comparison theorem for spectral sequences, Proc. Cambridge Philos. Soc. 53 (1957), 57-62.

CORNELl UNIVERSTTY, ITHACA, NEW YORK 